Supplement of Atmos. Chem. Phys., 21, 8003-8021, 2021

https://doi.org/10.5194/acp-21-8003-2021-supplement

(C) Author(s) 2021. CC BY 4.0 License.

(c) (1)

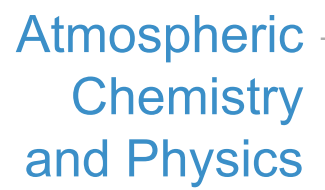

Gov

Supplement of

\title{
Analysis of secondary organic aerosol simulation bias in the Community Earth System Model (CESM2.1)
}

Yaman Liu et al.

Correspondence to: Xinyi Dong (dongxy@nju.edu.cn)

The copyright of individual parts of the supplement might differ from the article licence. 


\begin{tabular}{|c|c|c|c|}
\hline & Reactants & Products & Rate Constant \\
\hline 1 & ISOP + OH & $\begin{array}{c}\mathrm{ISOP}+\mathrm{OH}+0.0031 * \mathrm{SOAG} 0+0.0035 * \mathrm{SOAG} 1+ \\
0.0003 * \mathrm{SOAG} 2+0.0271 * \mathrm{SOAG} 3+0.0474 * \mathrm{SOAG} 4\end{array}$ & $2.54 \mathrm{e}-11 \exp (410 / \mathrm{T})$ \\
\hline 2 & $\mathbf{I S O P}+\mathbf{O 3}$ & $\mathrm{ISOP}+\mathrm{O} 3+0.0033 * \mathrm{SOAG} 3$ & $1.05 \mathrm{e}-14 \exp (-2000 / \mathrm{T})$ \\
\hline 3 & ISOP + NO3 & $\mathrm{ISOP}+\mathrm{NO} 3+0.059024 * \mathrm{SOAG} 3+0.025024 * \mathrm{SOAG} 4$ & $3.03 e-12 \exp (-446 / T)$ \\
\hline 4 & MTERP + NO3 & $\mathrm{MTERP}+\mathrm{NO} 3+0.17493 * \mathrm{SOAG} 3+0.59019 * \mathrm{SOAG} 4$ & $1.20 \mathrm{e}-12 \exp (490 / \mathrm{T})$ \\
\hline 5 & MTERP + O3 & $\begin{array}{l}\text { MTERP + O3 + 0.0508*SOAG0 + 0.1149*SOAG } 1+ \\
0.0348 * \text { SOAG2 + 0.0554*SOAG } 3+0.1278 * \text { SOAG } 4\end{array}$ & $6.30 \mathrm{e}-16 \exp (-580 / \mathrm{T})$ \\
\hline 6 & MTERP + OH & $\begin{array}{l}\text { MTERP }+\mathrm{OH}+0.0508 * \mathrm{SOAG} 0+0.1149 * \mathrm{SOAG} 1+ \\
0.0348 * \mathrm{SOAG} 2+0.0554 * \mathrm{SOAG} 3+0.1278 * \mathrm{SOAG} 4\end{array}$ & $1.20 \mathrm{e}-11 \exp (440 / \mathrm{T})$ \\
\hline 7 & BCARY + NO3 & $\mathrm{BCARY}+\mathrm{NO} 3+0.17493 * \mathrm{SOAG} 3+0.59019 * \mathrm{SOAG} 4$ & $1.900 \mathrm{e}-11$ \\
\hline 8 & BCARY + O3 & $\begin{array}{c}\mathrm{BCARY}+\mathrm{O} 3+0.2202 * \mathrm{SOAG} 0+0.2067 * \mathrm{SOAG} 1+ \\
0.0653 * \mathrm{SOAG} 2+0.1284 * \mathrm{SOAG} 3+0.114 * \mathrm{SOAG} 4\end{array}$ & $1.200 \mathrm{e}-14$ \\
\hline 9 & BCARY + OH & $\begin{array}{c}\mathrm{BCARY}+\mathrm{OH}+0.2202 * \mathrm{SOAG} 0+0.2067 * \mathrm{SOAG} 1+ \\
0.0653 * \mathrm{SOAG} 2+0.1284 * \mathrm{SOAG} 3+0.114 * \mathrm{SOAG} 4\end{array}$ & $2.000 \mathrm{e}-10$ \\
\hline 10 & BENZENE + OH & $\begin{array}{l}\mathrm{BENZENE}+\mathrm{OH}+0.0023 * \mathrm{SOAG} 0+0.0008 * \mathrm{SOAG} 1 \\
+0.0843 * \mathrm{SOAG} 2+0.0443 * \mathrm{SOAG} 3+0.1621 * \mathrm{SOAG} 4\end{array}$ & $2.30 \mathrm{e}-12 \exp (-193 / \mathrm{T})$ \\
\hline 11 & TOLUENE + OH & $\begin{array}{l}\text { TOLUENE }+\mathrm{OH}+0.1364 * \mathrm{SOAG} 0+0.0101 * \mathrm{SOAG} 1 \\
+0.0763 * \mathrm{SOAG} 2+0.2157 * \mathrm{SOAG} 3+0.0232 * \mathrm{SOAG} 4\end{array}$ & $1.70 \mathrm{e}-12 \exp (352 / \mathrm{T})$ \\
\hline 12 & XYLENES + OH & $\begin{array}{l}\text { XYLENES }+\mathrm{OH}+0.1677 * \mathrm{SOAG} 0+0.0174 * \mathrm{SOAG} 1 \\
+0.086 * \mathrm{SOAG} 2+0.0512 * \mathrm{SOAG} 3+0.1598 * \mathrm{SOAG} 4\end{array}$ & $1.700 \mathrm{e}-11$ \\
\hline 13 & $\mathbf{I V O C}+\mathbf{O H}$ & $\begin{array}{c}\mathrm{OH}+0.2381 * \mathrm{SOAG} 0+0.1308 * \mathrm{SOAG} 1+ \\
0.0348 * \mathrm{SOAG} 2+0.0076 * \mathrm{SOAG} 3+0.0113 * \mathrm{SOAG} 4\end{array}$ & $1.340 \mathrm{e}-11$ \\
\hline 14 & $\mathbf{S V O C}+\mathbf{O H}$ & $\begin{array}{c}\mathrm{OH}+0.5931 * \mathrm{SOAG} 0+0.1534 * \mathrm{SOAG} 1+ \\
0.0459 * \mathrm{SOAG} 2+0.0085 * \mathrm{SOAG} 3+0.0128 * \mathrm{SOAG} 4\end{array}$ & $1.340 \mathrm{e}-11$ \\
\hline 15 & GLYOXAL + aer & SOAG0 & $\mathrm{f}(\mathrm{SAD}), \gamma=0.0002^{\mathrm{b}}$ \\
\hline
\end{tabular}

a Emmons et al. (2020)

${ }^{b}$ Function of aerosol surface area density (SAD), see Emmons et al. (2020) for details 
Table S2. Chemical formula and description of species in Table S1(Emmons et al., 2020).

\begin{tabular}{|c|c|c|}
\hline Species & Chemical Formula & Description \\
\hline ISOP & $\mathrm{C} 5 \mathrm{H} 8$ & isoprene \\
\hline MTERP & $\mathrm{C} 10 \mathrm{H} 16$ & lumped monoterpenes \\
\hline BCARY & $\mathrm{C} 15 \mathrm{H} 24$ & beta-caryophyllene and other sesquiterpenes \\
\hline BENZENE & C6H6 & benzene \\
\hline TOLUENE & $\mathrm{C} 7 \mathrm{H} 8$ & toluene \\
\hline XYLENES & $\mathrm{C} 8 \mathrm{H} 10$ & lumped xylenes \\
\hline IVOC & $\mathrm{C} 13 \mathrm{H} 28$ & intermediate volatility organic precursor of VBS SOA \\
\hline SVOC & $\mathrm{C} 22 \mathrm{H} 46$ & semi-volatile organic precursor of VBS SOA \\
\hline GLYOXAL & $\mathrm{C} 2 \mathrm{H} 2 \mathrm{O} 2$ & glyoxal \\
\hline $\mathbf{O H}$ & $\mathrm{OH}$ & hydroxyl radical \\
\hline $\mathbf{O 3}$ & $\mathrm{O} 3$ & ozone \\
\hline NO3 & NO3 & nitrate radical \\
\hline SOAG0 & $\mathrm{C} 15 \mathrm{H} 38 \mathrm{O} 2$ & SOA gas-phase precursor VBS bin 0 (mol.wt. $=250 \mathrm{~g} / \mathrm{mol})$ \\
\hline & & (Shrivastava et al., 2015) \\
\hline SOAG1 & $\mathrm{C} 15 \mathrm{H} 38 \mathrm{O} 2$ & SOA gas-phase precursor VBS bin 1 \\
\hline SOAG2 & $\mathrm{C} 15 \mathrm{H} 38 \mathrm{O} 2$ & SOA gas-phase precursor VBS bin 2 \\
\hline SOAG3 & $\mathrm{C} 15 \mathrm{H} 38 \mathrm{O} 2$ & SOA gas-phase precursor VBS bin 3 \\
\hline SOAG4 & $\mathrm{C} 15 \mathrm{H} 38 \mathrm{O} 2$ & SOA gas-phase precursor VBS bin 4 \\
\hline
\end{tabular}


Table S3. The average OA of four ATom campaigns (Wofsy et al., 2018) and CAM-Chem-SD; the mean bias (MB), normalized mean bias (NMB), normalized mean error (NME), root mean square error (RMSE) and correlation coefficient (CC) between campaigns and CAMChem-SD.

\begin{tabular}{cccccccc}
\hline Aircraft & Mean Obs. & Mean Sim. & MB & NMB(\%) & NME(\%) & RMSE & CC \\
\hline ATom1 & 0.38 & 0.26 & -0.12 & -29.6 & 64.1 & 0.70 & 0.72 \\
ATom2 & 0.16 & 0.10 & -0.06 & -39.6 & 76.8 & 0.26 & 0.58 \\
ATom3 & 0.28 & 0.13 & -0.15 & -54.7 & 76.5 & 0.61 & 0.40 \\
ATom4 & 0.38 & 0.11 & -0.27 & -70.8 & 81.2 & 0.90 & 0.33 \\
\hline
\end{tabular}




\section{surface POA emission (molecules $/ \mathrm{cm}^{2} / \mathrm{s}$ )}

(a) CONUS

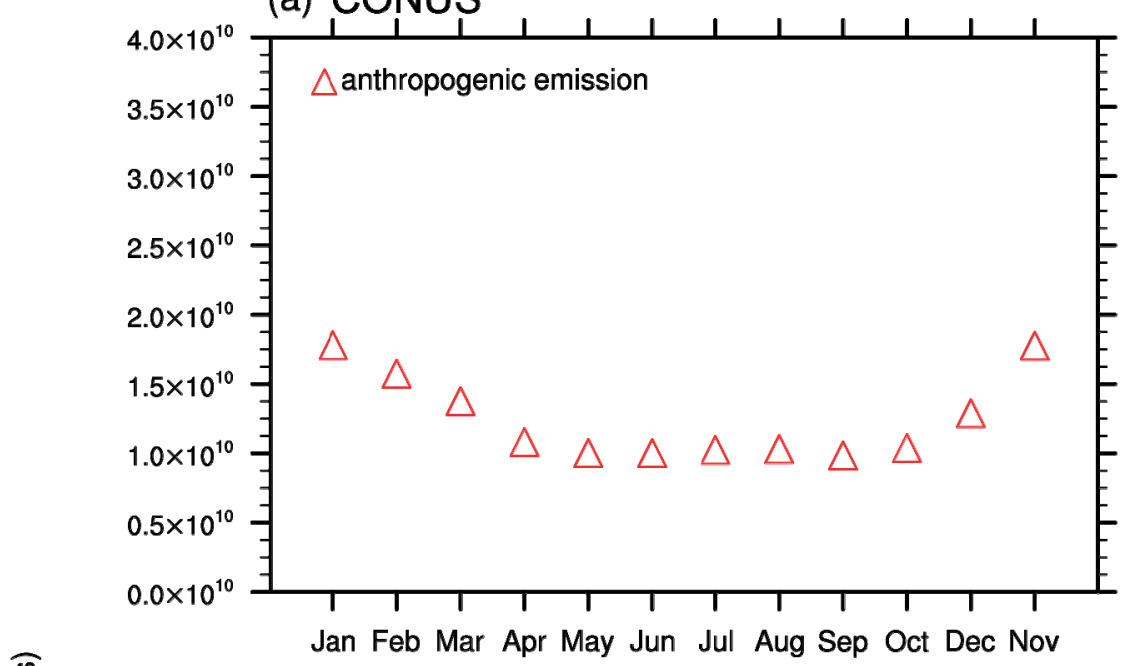

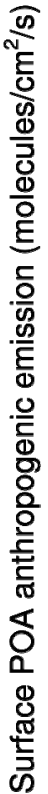

(b) EUS

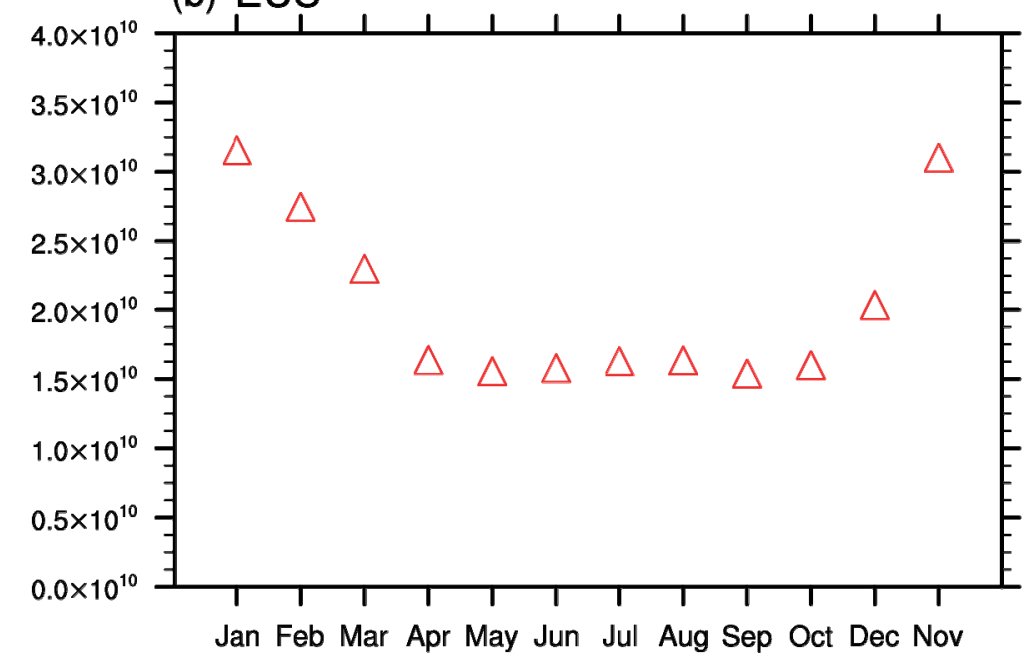

(c) WUS

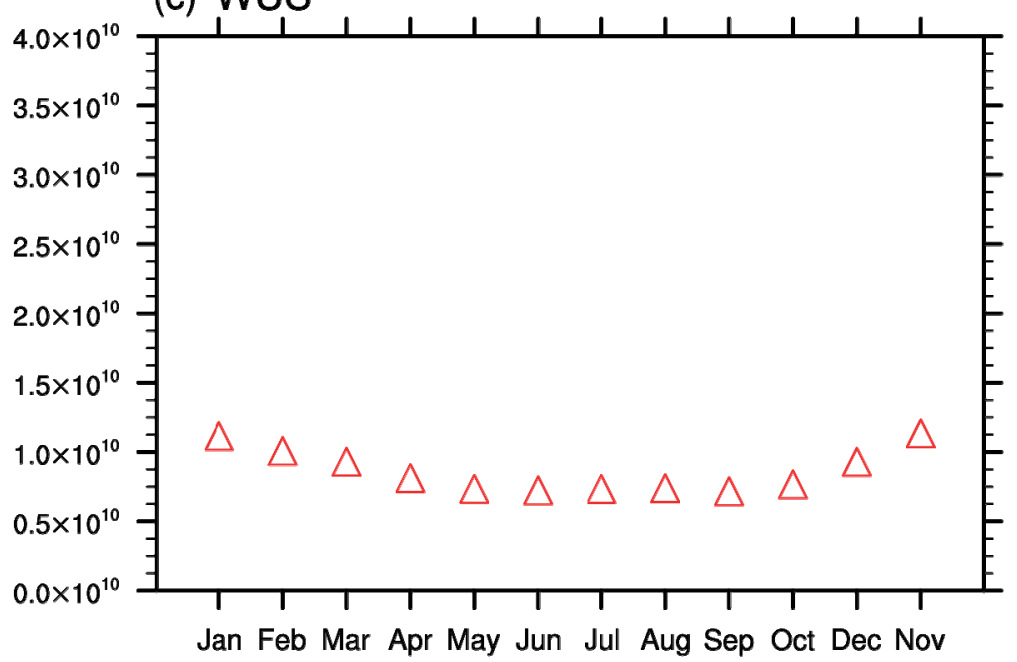

month

Figure. S1. seasonal cycle of 1988-2019 average surface Primary Organic Aerosol (POA) anthropogenic emission used for CAM-ChemSD (red upper triangles) over CONUS (a), EUS (b) and WUS (c). 
(a) aircraft trajectories of ATom
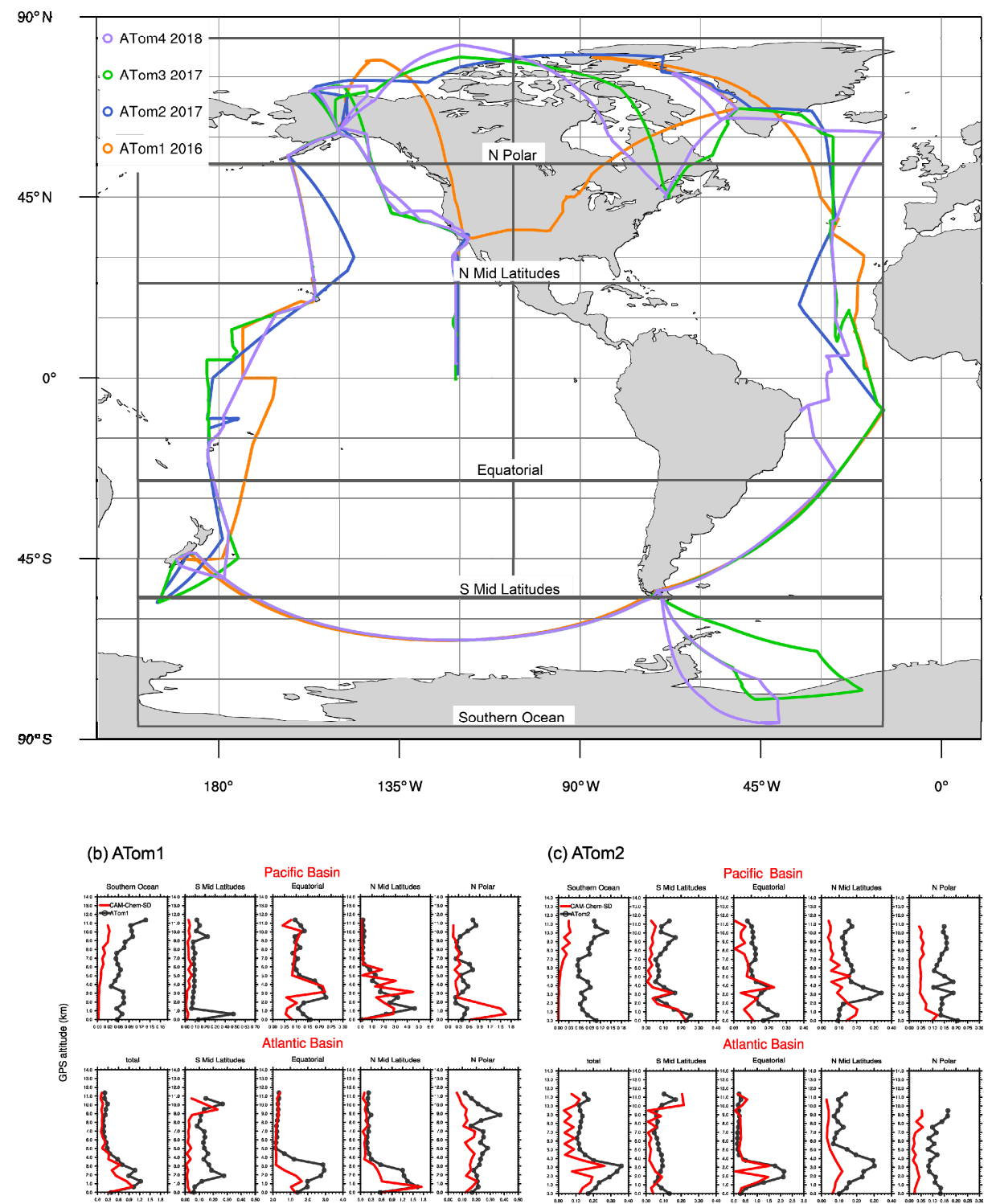

(c) ATom2
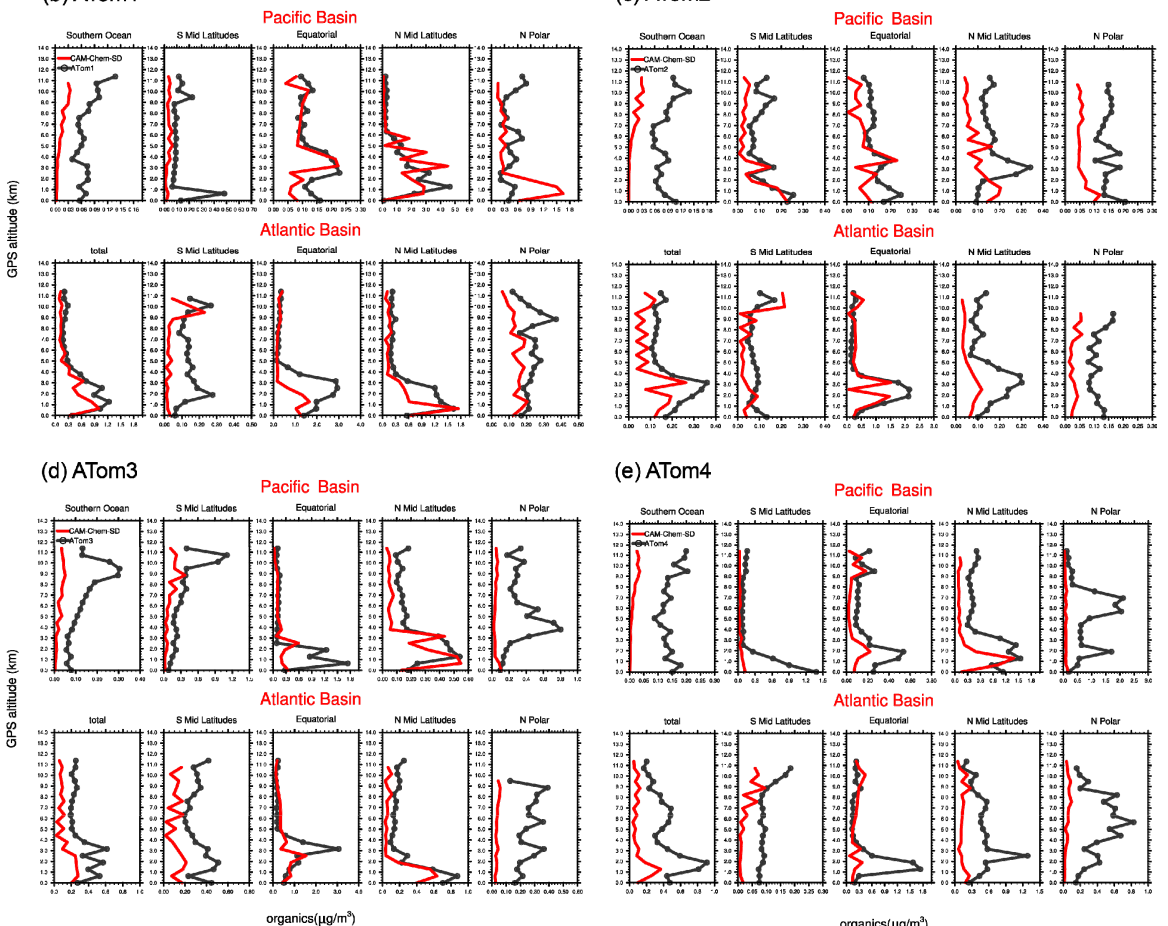

(e) ATom4
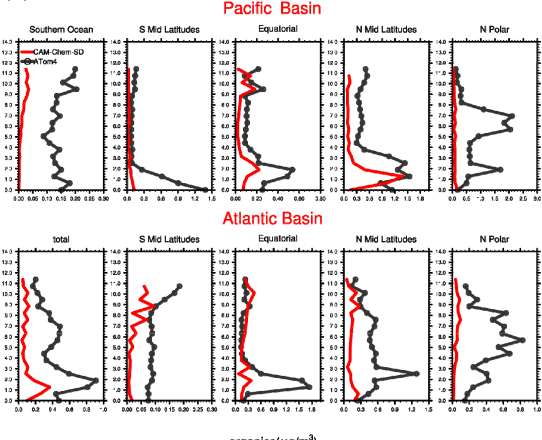

Figure. S2. Validation against ATom flight. (a) trajectories of four ATom flights; subdomains divisions are shown in grey boxes. Vertical profile of organics concentration of CAM-Chem-SD (red lines) and flight measurements (black marker lines) during ATom1 (b), ATom2 (c), ATom3 (d) and ATom4 (e) campaigns. 


\section{July US average SOA concontration}

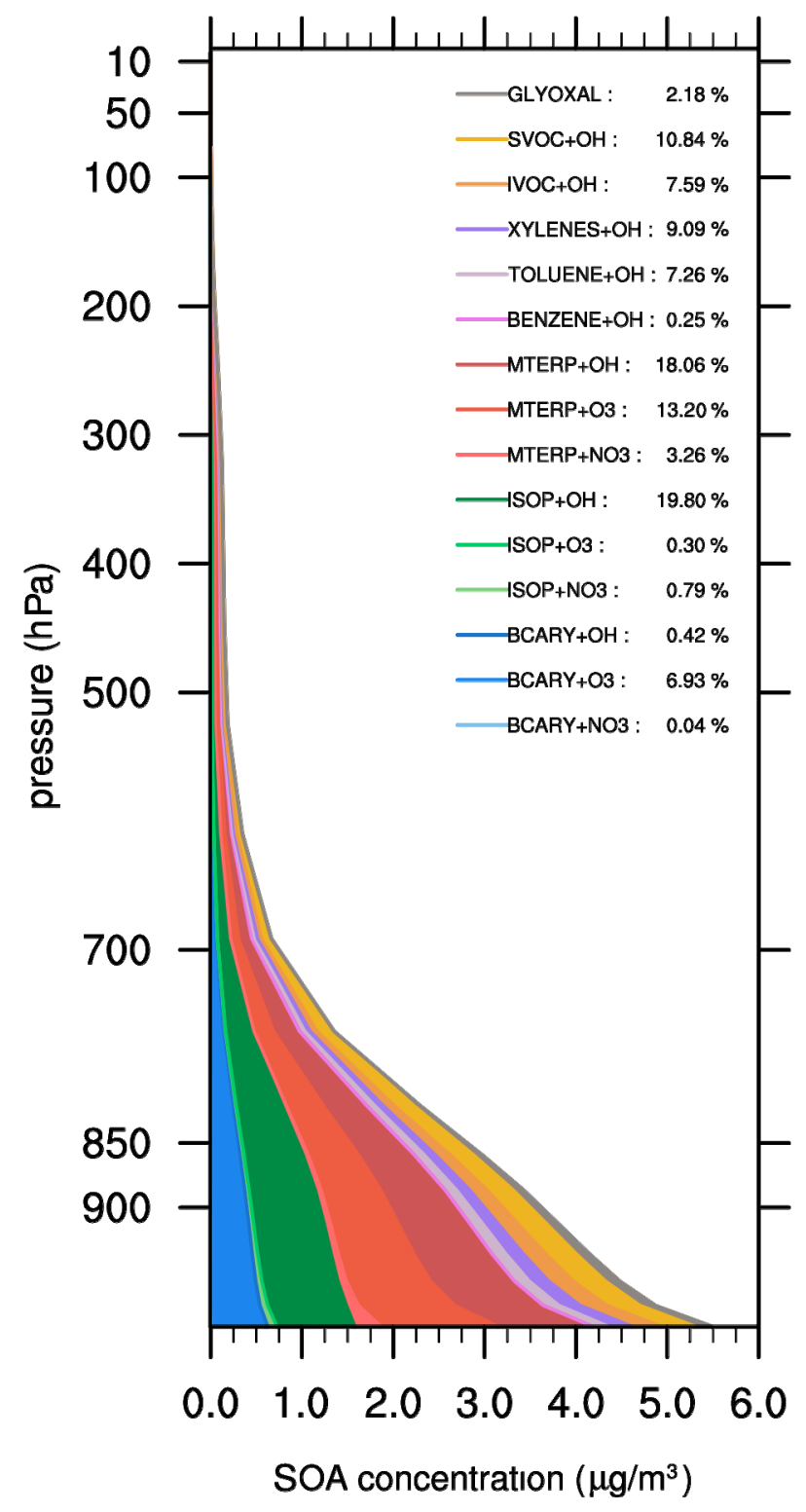

Figure. S3. Vertical profile of SOA formed by 15 reactions over US in July, 2010. The vertical average relative contribution of each reaction is shown as the number in the legend. 
relationship between OA bias and VOCs flux of CAM-Chem-SD
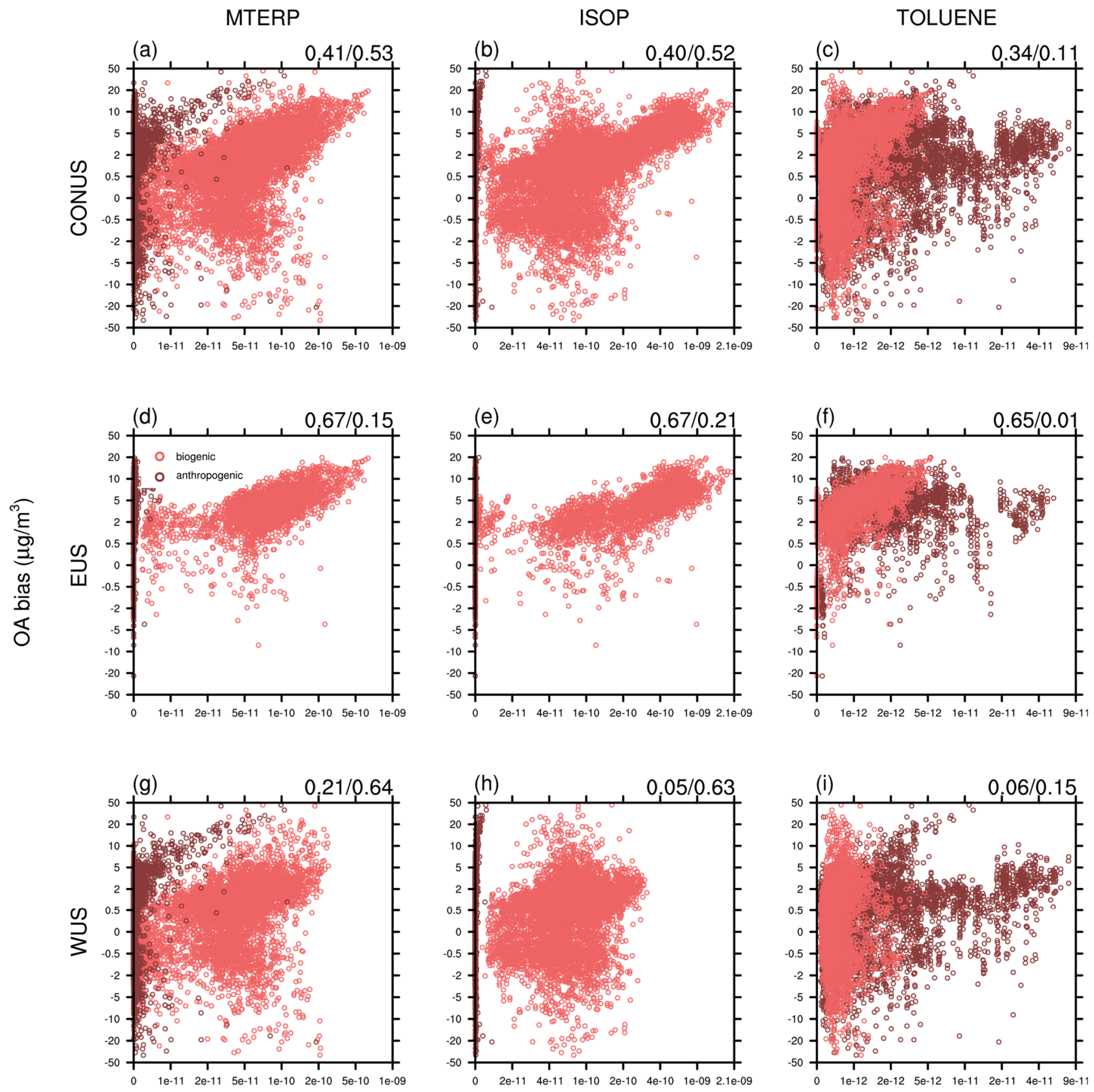

surface VOCs flux $\left(\mathrm{kg} / \mathrm{m}^{2} / \mathrm{s}\right)$

Figure. S4. The relationship between surface OA bias and MTERP (panel a, d, g), ISOP (panel b, e, h), TOLUENE (panel c, f, i) flux in the summer of 1988 to 2019 over CONUS (panel a $\sim$ c), EUS (panel d f) and WUS (panel g $\sim$ i). Surface VOCs flux are split into biogenic emission flux from MEGAN (light red dots) and other flux referred as anthropogenic flux (dark red dots). The numbers shown in each panel are the correlation coefficient between $\mathrm{OA}$ bias and biogenic flux, followed by the correlation coefficient between $\mathrm{OA}$ bias and anthropogenic flux. 


\section{surface OA concentration $\left(\mu \mathrm{g} / \mathrm{m}^{3}\right)$}
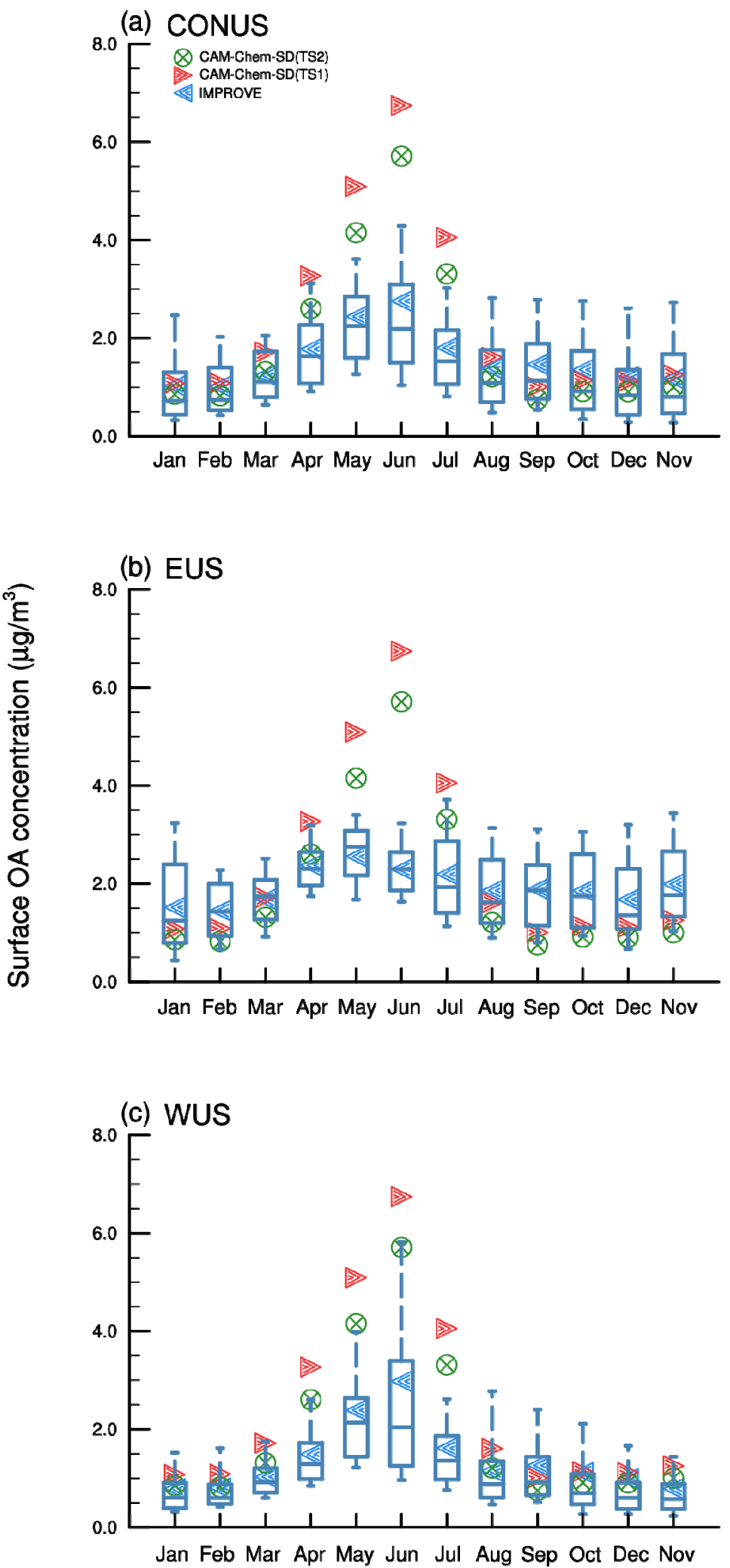

Figure. S5. 2013.03-2014.02 CONUS surface OA concentration of IMPROVE (blue dots), CAM-Chem-SD with MOZART-TS1 chemistry (red dots) and CAM-Chem-SD with MOZART-TS2 chemistry (green dots). Every blue box denotes the $10^{\text {th }}$, the $25^{\text {th }}$, the median, the $\mathbf{7 5}^{\text {th }}$ and the $90^{\text {th }}$ percentiles of the observations for all selected sites in each month. 


\section{Reference:}

Emmons, L. K., Schwantes, R. H., Orlando, J. J., Tyndall, G., Kinnison, D., Lamarque, J. F., Marsh, D., Mills, M. J., Tilmes, S., Bardeen, C., Buchholz, R. R., Conley, A., Gettelman, A., Garcia, R., Simpson, I., Blake, D. R., Meinardi, S., and Pétron, G.: The Chemistry Mechanism in the Community Earth System Model Version 2 (CESM2), Journal of Advances in Modeling Earth Systems, 12, 10.1029/2019ms001882, 2020.

Shrivastava, M., Easter, R. C., Liu, X., Zelenyuk, A., Singh, B., Zhang, K., Ma, P.-L., Chand, D., Ghan, S., Jimenez, J. L., Zhang, Q., Fast, J., Rasch, P. J., and Tiitta, P.: Global transformation and fate of SOA: Implications of lowvolatility SOA and gas-phase fragmentation reactions, Journal of Geophysical Research: Atmospheres, 120, 41694195, 10.1002/2014jd022563, 2015.

Wofsy, S. C., Afshar, S., Allen, H. M., Apel, E. C., Asher, E. C., Barletta, B., Bent, J., Bian, H., Biggs, B. C., Blake, D. R., Blake, N., Bourgeois, I., Brock, C. A., Brune, W. H., Budney, J. W., Bui, T. P., Butler, A., Campuzano-Jost, P., Chang, C. S., Chin, M., Commane, R., Correa, G., Crounse, J. D., Cullis, P. D., Daube, B. C., Day, D. A., Dean-Day, J. M., Dibb, J. E., DiGangi, J. P., Diskin, G. S., Dollner, M., Elkins, J. W., Erdesz, F., Fiore, A. M., Flynn, C. M., Froyd, K. D., Gesler, D. W., Hall, S. R., Hanisco, T. F., Hannun, R. A., Hills, A. J., Hintsa, E. J., Hoffman, A., Hornbrook, R. S., Huey, L. G., Hughes, S., Jimenez, J. L., Johnson, B. J., Katich, J. M., Keeling, R. F., Kim, M. J., Kupc, A., Lait, L. R., Lamarque, J. F., Liu, J., McKain, K., McLaughlin, R. J., Meinardi, S., Miller, D. O., Montzka, S. A., Moore, F. L., Morgan, E. J., Murphy, D. M., Murray, L. T., Nault, B. A., Neuman, J. A., Newman, P. A., Nicely, J. M., Pan, X., Paplawsky, W., Peischl, J., Prather, M. J., Price, D. J., Ray, E. A., Reeves, J. M., Richardson, M., Rollins, A. W., Rosenlof, K. H., Ryerson, T. B., Scheuer, E., Schill, G. P., Schroder, J. C., Schwarz, J. P., St.Clair, J. M., Steenrod, S. D., Stephens, B. B., Strode, S. A., Sweeney, C., Tanner, D., Teng, A. P., Thames, A. B., Thompson, C. R., Ullmann, K., Veres, P. R., Vieznor, N., Wagner, N. L., Watt, A., Weber, R., Weinzierl, B., Wennberg, P. O., Williamson, C. J., Wilson, J. C., Wolfe, G. M., Woods, C. T., and Zeng, L. H.: ATom: Merged Atmospheric Chemistry, Trace Gases, and Aerosols, in, ORNL Distributed Active Archive Center, 2018. 